\title{
Estudio del Mecanismo de Remoción de Hierro y Cobre presentes en Aceites Lubricantes Usados Mediante Bioadsorción
}

\author{
Albina S. Moreira, Márcia M. L. Duarte, Júlio Nandenha y Gorete R. Macedo \\ Universidad Federal do Rio Grande do Norte, Departamento de Engenharia Química, Centro de \\ Tecnologia, Av. Senador Salgado Filho 3000, Campus Universitário, 59072-970 Natal, RN-Brasil \\ (e-mail: albina@eq.ufrn.br,marcia@eq.ufrn.br, jnandenha@gmail.com,gomacedo@eq.ufrn.br)
}

\begin{abstract}
Resumen
En este trabajo se utilizó como biomasa el alga marina Sargassum sp. para estudiar la influencia de la velocidad de agitación, la temperatura y la concentración inicial de biomasa en la remoción de hierro y cobre presentes en aceites lubricantes usados. Se realizó un diseño experimental y un estudio cinético. Los experimentos fueron llevados a cabo con muestras de aceites lubricantes usados y cantidades pre-determinadas de algas durante tiempo suficiente para que la mezcla alcance el equilibrio. A partir de los resultados obtenidos se observó que en las condiciones estudiadas, a medida que la cantidad de biomasa aumenta es menor la capacidad de adsorción del hierro y del cobre, probablemente debido a una disminución del área interfacial de contacto. El estudio muestra que es posible obtener una función que muestre el nivel de influencia de cada una de las variables del sistema.
\end{abstract}

Palabras clave: aceite lubricante usado, bioadsorción, sargassum sp., remoción de metales

\section{Study of the Removal Mechanism of Iron and Copper from Used Lubricant Oils trough Bioadsorption}

\begin{abstract}
In this study biomass of the marine alga Sargassum sp was used to study the influence of agitation velocity, temperature and initial biomass concentration on the removal of iron and copper from used lubricant oils. An experimental design and a kinetic study were done. The experiments were carried out using samples of used lubricant oils and predetermined amounts of algae allowing sufficient time for the mixture to reach equilibrium conditions. The results showed that, under the conditions studied, the larger the amount of biomass present, the lower the adsorption capacity of iron and copper, probably due to a decrease in interfacial contact area. The study shows that it is possible to obtain a function that represents the influence of each of the variables that shows the degree of influence of each of the system variables.
\end{abstract}

Keywords: used lubricating oil, bioadsorption, sargassum sp., metal removal 


\section{INTRODUCCIÓN}

El acelerado desarrollo industrial lleva a la comunidad científica a buscar nuevas tecnologías de prevención y control de la contaminación, intentado ofrecer al hombre la posibilidad del uso o recuperación de los recursos naturales. En Brasil existe un déficit en el tratamiento de efluentes provocando un gran impacto sobre el medio ambiente, a la salud y también a la calidad de vida de la población. Por esto existe una necesidad de investigar y aplicar técnicas de bajo costo que puedan ser usadas en la implantación de tratamientos de residuos líquidos y sólidos (Barros et al., 2001).

La contaminación del agua, aire y suelo por metales pesados es uno de los problemas ambientales más severos, y es muy difícil de resolver. Las fuentes más comunes de contaminación por dichos metales son: los procesos de petróleo, las plantas generadoras de energía y los procesos metalúrgicos (Acosta et al., 2007).

Se tiene también que la ingestión de algunos metales, por ejemplo el cobre, por el hombre en valores superiores a los recomendados puede causar: irritación y corrosión de la mucosa gástrica, daño en la estructura capilar, en los riñones, en el sistema nervioso seguido por depresión y también se puede acumular en el hígado (Gupta y Torres, 1998; Alloway y Ayres, 1994).

Se ha demostrado la capacidad de ciertos microorganismos para concentrar los metales, por lo que durante las últimas décadas han sido usados como una alternativa para la remoción de metales del medio ambiente a través del proceso de bioadsorción. El término "bioadsorción" es definido como un proceso en el cual los sólidos de origen natural o sus derivados son usados en la retención de metales pesados de un ambiente acuoso.

Diferentes estudios se han realizado para evaluar el potencial de una variedad de materiales biológicos para eliminar los metales pesados de residuos industriales. Las algas, sus materiales derivados, turbas, musgos, chitosan, lignina, algunos tipos de bacterias vivas y muertas, hongos y residuos agrícolas, entre otros; han sido usados como adsorbentes en pruebas de bioadsorción (Volesky, 1990; Bailey et al., 1999).

El proceso de bioadsorción de los metales pesados por algas marinas pretratadas es una excelente opción para la descontaminación de distintos efluentes acuosos (Figueira et al., 2004; Kratochvil y Volesky, 2000; Schmitt et al., 2001), debido a esto se decidió estudiar el proceso en un medio orgánico con la finalidad de remover algunos metales pesados presentes en los aceites lubricantes usados. Esta aplicación es una innovación tecnológica, ya que en la literatura son solamente encontrados estudios aplicados para sistemas acuosos.

\section{MATERIALES Y MÉTODOS}

En este trabajo se utilizó un aceite lubricante automotor usado, conocido como Rimula-D-Shell SAE 40, que fue aportado por una compañía de autobús urbana que opera en la ciudad de Natal (Río Grande do Norte, Brasil). El material bioadsorbente consistió en la especie de alga marina Phaeophyceae (feofitas - pardas) del género Sargassum sp, que fue recogida en la orilla de la playa "Areia Preta" localizada en la ciudad de Natal-RN/Brasil, durante el mes de Abril de 2004.

\section{Caracterización del aceite lubricante que tiene la presencia de metales pesados}

Para realizar la caraterización se realizó una combustión de las diferentes muestras de aceite. Después se llevó a cabo una acidificación hasta una completa solubilización de las cenizas. Los restos fueron caracterizados por medio de espectrometría de absorción atómica (FAAS), usando un "Varian AA 12/1475 Gemini Spectrometer", para determinar el tipo y concentración de cualquier metal pesado presente en el aceite.

\section{Material Bioadsorbente}

La alga marina Sargassum sp. fue usada como material biosadorbente bajo dos condiciones diferentes: noprotonada y protonada con tratamiento de $\mathrm{HCl}$. La protonación se realizó con el 
propósito de retirar los metales alcalinos como $\mathrm{Na}$, $\mathrm{Ca}$ y $\mathrm{Mg}$ contenidos en la estructura de las células. Este paso consistió en el lavado de $15 \mathrm{~g}$ de alga marina seca (secada a $60{ }^{\circ} \mathrm{C}$ por 12 horas) con $400 \mathrm{~cm}^{3}$ de una solución $0,06 \mathrm{M}$ de $\mathrm{HCl}$ durante 3 horas, a una temperatura del ambiente aproximadamente constante (entre $25-28{ }^{\circ} \mathrm{C}$ ). Luego, la muestra de alga marina se lavó completamente con agua destilada hasta que la solución lavada alcanzó un $\mathrm{pH}$ de 4,5; en este punto la muestra se secó de nuevo a $60^{\circ} \mathrm{C}$ durante un período de 12 horas (Hayashi, 2001). Después del secado final, la muestra fue molida utilizando un mortero y fue guardada en un recipiente hermético para evitar el contacto con el aire, debido a su alta propiedad higroscópica.

\section{Experimentos de Bioadsorción}

La metodología usada en los ensayos experimentales consistió en el uso de matraces Erlenmeyer de $1000 \mathrm{~cm}^{3}$ cada uno, que contenían $500 \mathrm{~cm}^{3}$ de aceite lubricante usado y 0,5 g de biomasa. Los ensayos se realizaron a una temperatura constante de $25^{\circ} \mathrm{C}$. La biomasa se mezcló uniformemente y en forma constante con el aceite lubricante con un agitador rotatório que opera a $200 \mathrm{rpm}$. Las mismas condiciones fueron aplicadas a otros materiales bioadsorbentes: protonado (previamente tratado con $\mathrm{HCl}$ ) y no protonado. Todos los experimentos se llevaron a cabo en duplicado y los valores promedios fueron los usados en los análisis de los resultados.

La cantidad de hierro o cobre adsorbida por la biomasa (q) y la eficiencia de bioadsorción (E) puede calcularse usando las ecuaciones 1 y 2 , respectivamente:

$$
\begin{aligned}
& q=\left(\frac{C_{i}-C_{f}}{m}\right) * V \\
& E=\left(\frac{C_{i}-C_{f}}{C_{i}}\right) * 100
\end{aligned}
$$

Donde:

$C_{i}$ y $C_{f}$ : Cantidades inicial y final de hierro o cobre, en la fase aceitosa, mg; m: Peso seco de biomasa, $\mathrm{g}$; V: Volumen de aceite, $\mathrm{cm}^{3}$.

Se utilizó la ecuación 1 para calcular la cinética y el efecto de la cantidad de biomassa y la ecuación 2 para el cálculo de la eficiencia en el diseño experimental.

\section{Cinética de la bioadsorción}

Para el estudió de la cinética de bioadsorción fueron retiradas las muestras de aceite en diferentes intervalos de tiempo, luego el contenido se filtró usando un filtro cuantitativo (de $12,5 \mathrm{~cm}$ de diámetro y con poros de $8 \mu \mathrm{m}$ ) y por último se analizó la concentración de cobre y hierro a través de un espectrómetro de absorción atómica.

\section{Efecto del contenido de biomasa}

Se estudio el efecto de la cantidad utilizada de biomasa durante 10 horas (fue el período de tiempo que exigió para lograr el equilibrio). Biomasa y metal libre fue empleado en el experimento de control. Al final de este procedimiento, la fase aceitosa ya estaba separada de la biomasa por el vacío.

Para evaluar la influencia de factores como: velocidad de agitación, temperatura, cantidad de biomasa (BM) y concentración de ácido clorhídrico en la protonación, fue utilizado un diseño factorial completo $2^{4}$ con triplicidad en el punto central. Los niveles estudiados se muestran en la Tabla 1. 
Tabla 1: Parámetros usados en el estudio del mecanismo de remoción de Fe y Cu

\begin{tabular}{|l|l|l|l|}
\hline \multirow{2}{*}{ Factores } & \multicolumn{3}{c|}{ Niveles } \\
\cline { 2 - 4 } & Mín. (-1) & $\begin{array}{l}\text { Central } \\
(0)\end{array}$ & $\begin{array}{l}\text { Máx. } \\
(+1)\end{array}$ \\
\hline $\begin{array}{l}\text { Velocidad de } \\
\text { agitación (AGIT) (rpm) }\end{array}$ & 200 & 300 & 400 \\
\hline Temperatura (TEMP) ( $\left.{ }^{\circ} \mathrm{C}\right)$ & 30 & 35 & 40 \\
\hline Cantidad de biomasa (BM) (g) & 0,20 & 0,60 & 1,00 \\
\hline $\begin{array}{l}\text { Concentración de HCl en la protonación (HCl) } \\
\text { (mol/L) }\end{array}$ & 0,06 & 0,08 & 0,10 \\
\hline
\end{tabular}

\section{RESULTADOS Y DISCUSIÓN}

La caracterización del aceite automotor usado consistió en la identificación del tipo de metal y de sus concentraciones iniciales. Los datos encontrados se muestran en la Tabla 2.

Tabla 2: Caracterización del aceite lubricante usado

\begin{tabular}{|c|c|}
\hline Elemento & Concentración $\left(\mathrm{mg} \cdot \mathrm{L}^{-1}\right)$ \\
\hline $\mathrm{Fe}$ & 43,95 \\
\hline $\mathrm{Cu}$ & 3,75 \\
\hline $\mathrm{Pb}$ & 1,92 \\
\hline $\mathrm{Ni}$ & 1,35 \\
\hline $\mathrm{Cr}$ & 1,25 \\
\hline
\end{tabular}

Es posible observar que el hierro se encontró en una concentración mayor en el aceite lubricante usado, seguido por el cobre, plomo, níquel y cromo. Fueron seleccionados el hierro y el cobre para los estudios debido a que sus concentraciones son las más altas.

La Tabla 3 muestra datos de los experimentos de bioadsorción con el material bioadsorbente protonado y no protonado, y la eficiencia de remoción de los metales pesados encontrados en el aceite.

Tabla 3: Concentración de metales después del proceso de bioadsorción.

\begin{tabular}{|c|c|c|c|c|}
\hline & Elemento & Conc. inicial $\left(\mathrm{mg} \cdot \mathrm{L}^{-1}\right)$ & Conc. final $\left(\mathrm{mg} \cdot \mathrm{L}^{-1}\right)$ & Removido (\%) \\
\hline \multirow{2}{*}{ Protonado } & $\mathrm{Fe}$ & 43,95 & 27,45 & 37,54 \\
\cline { 2 - 5 } & $\mathrm{Cu}$ & 3,75 & 2,56 & 31,73 \\
\hline \multirow{2}{*}{ No protonado } & $\mathrm{Fe}$ & 43,95 & 33,70 & 23,32 \\
\cline { 2 - 5 } & $\mathrm{Cu}$ & 3,75 & 2,95 & 21,33 \\
\hline
\end{tabular}

El análisis de los datos mostrados en la Tabla 3, confirma que es más efectivo la remoción de los metales usando algas Sargassum sp. protonadas. Como una consecuencia de la protonación, los metales más leves son retirados del material bioadorbente dónde ellos se encuentran para ser unidos químicamente a los grupos del carboxílico, hidroxilo y sulfatos de los polisacáridos que forman la composición celular (Hayashi, 2001).

Estos resultados indican que la protonación favorece la habilidad de bioadsorción de los iones de hierro y cobre. El tratamiento ácido mejoró la remoción de estos metales, probablemente debido a cambios producidos por la protonación en los pequeños sitios activos existentes. Además, la protonación reduce la competición por los sitios activos pequeños de los metales más leves, siendo eficazmente removidos por tal proceso. 
El estudio cinético del proceso de remoción de hierro y cobre por la alga Sargassum sp. se llevó a cabo con el propósito de seguir su progreso hasta alcanzar el equilibrio. Con la biomasa, los experimentos se realizaron en un agitador rotatório $\left(\mathrm{a} 25^{\circ} \mathrm{C}\right.$ y $\left.200 \mathrm{rpm}\right)$ usando $0,5 \mathrm{~g}$ de material biosadorbente y $500 \mathrm{~cm}^{3}$ de aceite lubricante (concentraciones iniciales para Fe y Cu de 43,95 y 3,75 $\mathrm{mg} . \mathrm{L}^{-1}$, respectivamente).

En las figuras 1 y 2, se muestran los resultados de las bioadsorciones de hierro y cobre en función del tiempo.

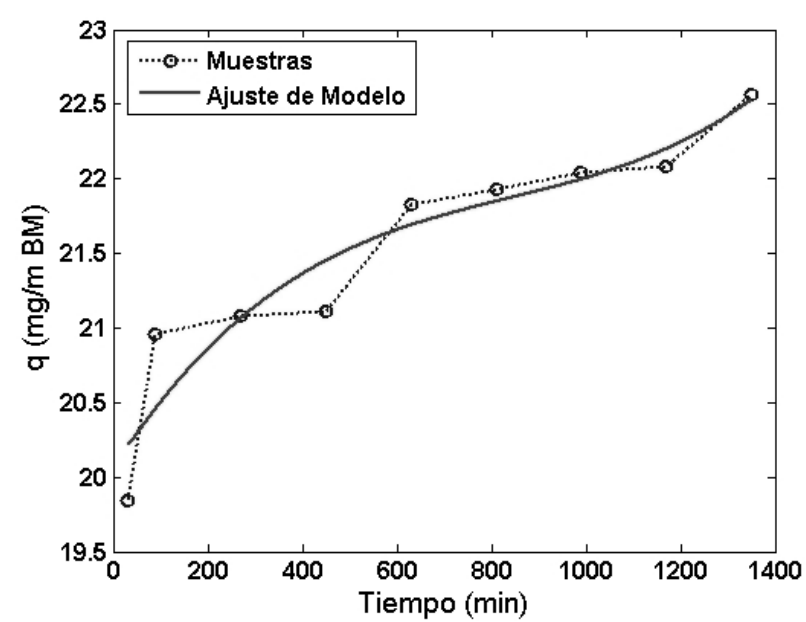

Fig. 1: Cinética de la bioadsorción de hierro por la Sargassum $s p$

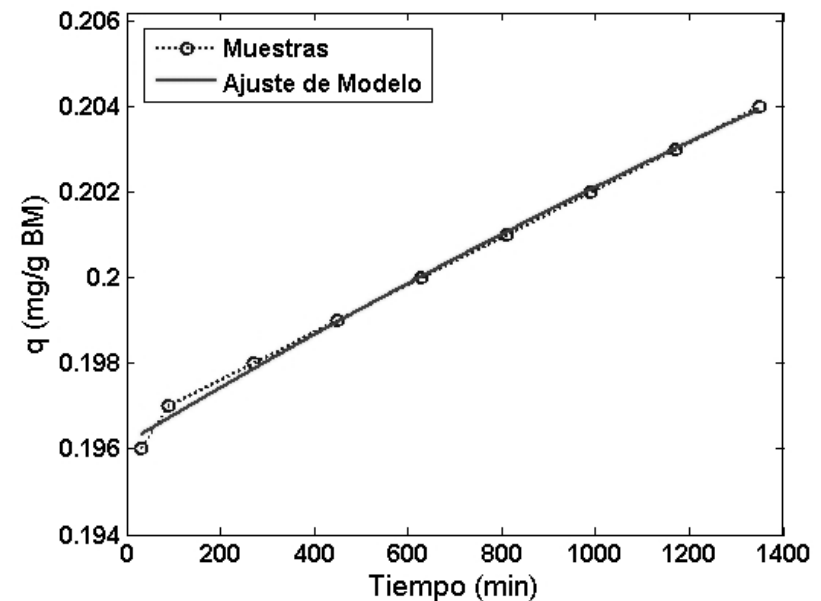

Fig. 2: Cinética de la bioadsorción de cobre por la Sargassum $s p$.

A partir de los datos experimentales obtenidos en el estudio cinético se realizó un ajuste de la curva utilizando el programa MATLAB (versión 7.0 release 14). Se pretendió obtener un modelo que prediga la variación de hierro y de cobre con el tiempo para el proceso de bioadsorción estudiado. De acuerdo con las figuras 1 y 2, las ecuaciones obtenidas se muestran a continuación:

Hierro:

$q=1,95 * 10^{-9} t^{3}-4,91 * 10^{-6} t^{2}+4,90 * 10^{-3} t+2,0$

Cobre:

$q=-5,77^{\star} 10^{-10} t-6,55^{\star} 10^{-6} t+0,20$

A partir de los datos experimentales, fueron obtenidos modelos polinómicos de tercer grado para el hierro y de segundo grado para el cobre.

Por las curvas de las figuras 1 y 2 se puede observar un buen ajuste del modelo tanto para el hierro como para el cobre. La desviación estándar, la variancia, el error promedio cuadrático y el promedio son mostrados en la Tabla 4.

Tabla 4: Datos estadísticos de los modelos ajustados

\begin{tabular}{|l|c|c|}
\hline \multirow{2}{*}{} & \multicolumn{2}{|c|}{ Metales } \\
\cline { 2 - 3 } & $\mathrm{Fe}$ & $\mathrm{Cu}$ \\
\hline Variancia & $6,8^{\star} 10^{-1}$ & $7,5^{\star} 10^{-6}$ \\
\hline Desviación estándar & $8,25^{\star} 10^{-1}$ & $2,74^{\star} 10^{-3}$ \\
\hline Error promedio cuadrático & $5,99^{\star} 10^{-2}$ & $2,46^{\star} 10^{-8}$ \\
\hline Promedio & 21,49 & 0,20 \\
\hline
\end{tabular}

Los datos experimentales de la Fig. 1 muestran que la concentración de iones férricos en la fase aceitosa se redujo en $11,32 \%$, lo que indica que el proceso de bioadsorción mejora con el tiempo de contacto y alcanza el equilibrio después de aproximadamente 10 horas. El período de tiempo 
requerido para el equilibrio entre las fases es una función de algunos factores como: el tipo de biomasa (la cantidad y tipo de sitios activos involucrados en el proceso), el tamaño y forma de la biomasa (activo o inactivo, libre o inmovilizada), además del material involucrado en el sistema de bioadsorción.

De forma similar al hierro, los resultados presentados en la Fig. 2 para el Cu, indican que la concentración del cobre en la fase aceitosa disminuye con el tiempo. El proceso del bioadsorción también mejora con el tiempo de contacto, no obstante en las condiciones experimentales aplicadas en estos experimentos, no se encontró evidencia de un punto de equilibrio (Fig. 2).

En la literatura se encontraron tiempos menores de captación cuando se trabajó en medio acuoso, con las biomasas Rhizopus arrhizus (Tobin et al., 1984) y M. rouxii (Yan y Viraraghavan, 2003). La bioadsorción de metales pesados por los componentes de la pared celular se basa en dos mecanismos: el de la captura por los grupos funcionales como carboxilatos, y el de la captación resultante de las interacciones físicoquimicas de los fenómenos de adsorción (Kappoor y Viraraghavar, 1995; Kratochvil y Volesky, 1998). Como en el sistema estudiado fue usado un medio acuoso el comportamiento es diferente, y esto puede ser atribuido a distintos factores entre los cuales puede ser mencionarse la viscosidad del aceite usado.

La capacidad de remoción (Eficiencia) del ión (hierro y cobre), también se ha evaluado como una función de la cantidad de biomasa, en gramos. Los resultados se muestran en las figuras 3 y 4.

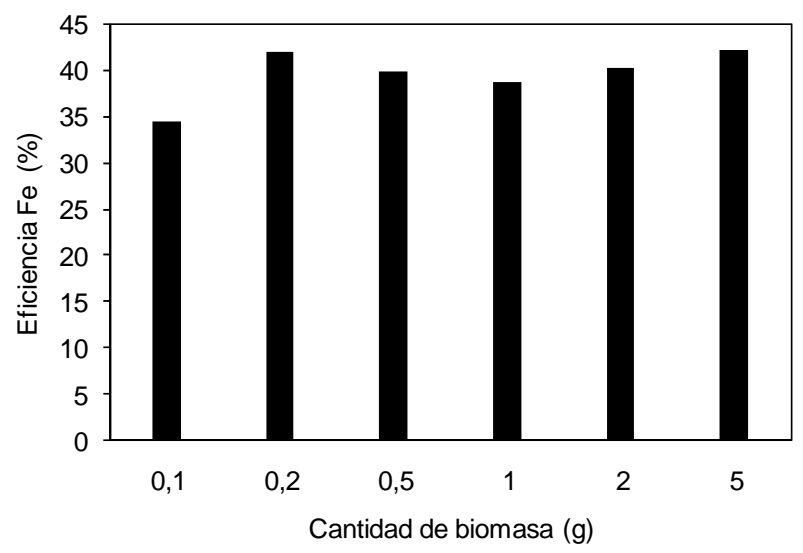

Fig. 3: Eficiencia de remoción de hierro como una función de la cantidad de biomasa.

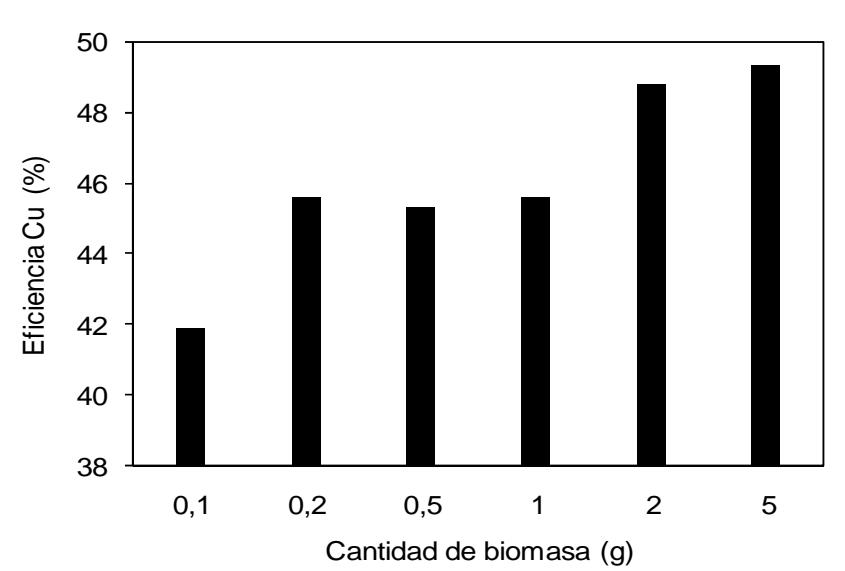

Fig. 4: Eficiencia de remoción de cobre como una función de la cantidad de biomasa.

Analizando la Fig. 3, se observa que la cantidad de biomasa no afecta el proceso de forma significativa y la eficiencia de remoción del hierro se mantiene practicamente constante, alrededor de $38 \%$, para la cantidad de biomasa analizada.

En la Fig. 4 muestra que la cantidad de biomasa mejora solamente la eficiencia de remoción de cobre con cantidades más elevadas de biomasa, por en cima de aproximadamente $2 \mathrm{~g}$.

Después de la evaluación del estudio cinético del mecanismo de bioadsorción se realizó un diseño experimental con el objetivo de estudiar los efectos de las variables: velocidad de agitación, temperatura, cantidad de biomasa (BM) y concentración de ácido clorhídrico en la protonación.

El análisis del diseño experimental fue realizado utilizándose el programa computacional "STATISTICA for Windows", (versión 6,0). Un estudio de la importancia estadística de las variables de entrada y sus interacciones en la respuesta fue realizado. Fueron evaluados los resultados de los efectos principales y de la interacción, las curvas de nivel y del diagrama de Pareto.

La Fig. 5, muestra una de las formas de evaluación de los efectos de las variables. El gráfico de Pareto, cuantifica los efectos de las variables estudiadas en la remoción del cobre. 
La magnitud de cada efecto está representada por las columnas y líneas transversales con un valor mayor o igual que $p=0,05$, lo que indica que el efecto de la variable o su interacción con las otras tiene un significado estadístico.

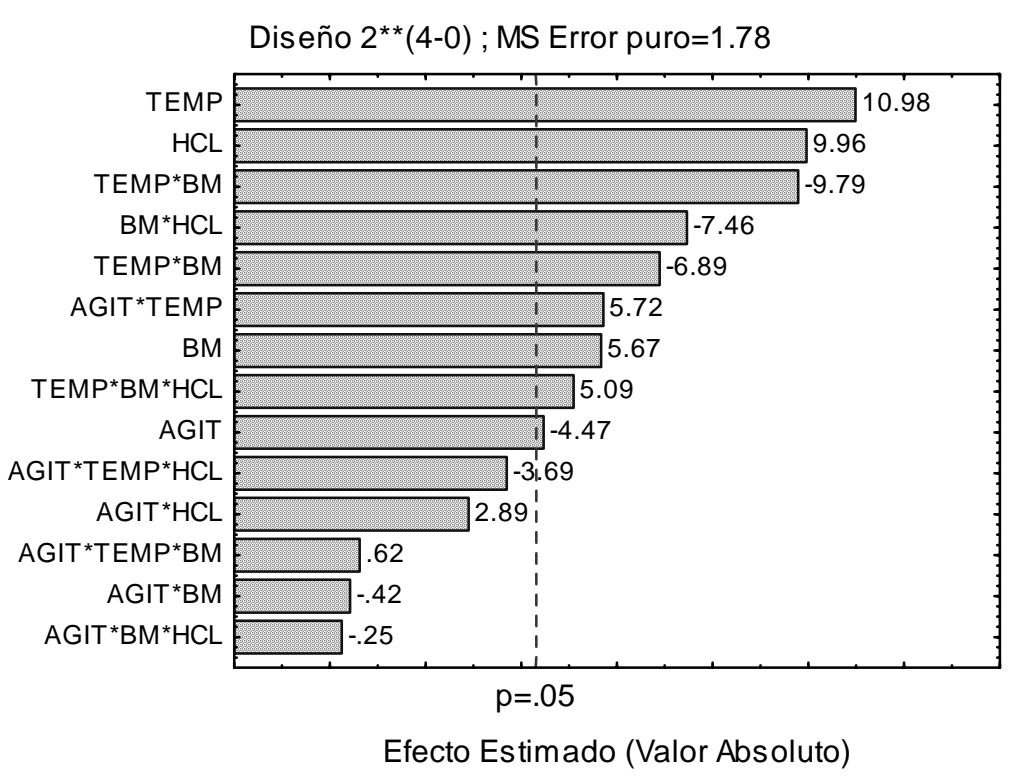

Fig. 5: Gráfico de Pareto para el estudio de la remoción del Cobre

En la Fig. 5, se observa que, para un nivel de confianza de 95\%, todos los factores estudiados fueron significativos en el proceso de remoción del metal, siendo la temperatura el factor más importante. También se puede observar que el efecto de la interacción entre los factores $T E M P^{\star} \mathrm{HCl}, \mathrm{BM}{ }^{*} \mathrm{HCl}$, $T E M{ }^{\star} B M, A G I T^{\star} T E M P$ e $T E M P^{\star} B M^{*} H C l$ también fueron significativos, solo que con una menor magnitud, comparada al efecto de la temperatura.

Los resultados experimentales obtenidos también fueron utilizados en la obtención de un modelo empírico, o sea una función polinómica, a través de la técnica de superficie de respuesta. El modelo fue evaluado comparando los valores calculados de los valores experimentales, según puede ser visto en la Fig. 6 . Se observa que los valores calculados se aproximan a los valores experimentales.

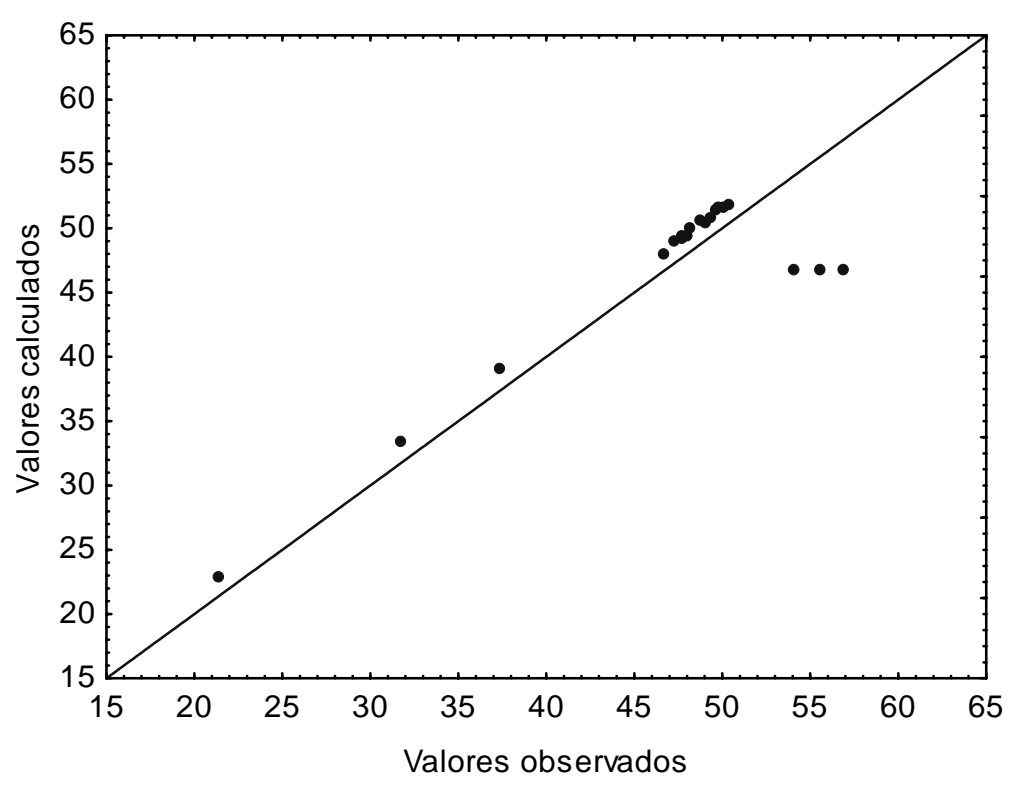

Fig. 6: Valores calculados versus valores experimentales para la remoción del cobre 
La capacidad de remoción (eficiencia) en función de la agitación y de la temperatura en la a remoción del cobre se muestra en la Fig. 7. Se puede observar que para altas temperaturas se obtienen las mayores eficiencias, con poca influencia de la agitación. A bajas temperaturas la influencia de agitación es mayor, observándose un aumento de la eficiencia con baja agitación. El aumento de la temperatura provoca una disminución de la viscosidad, lo que facilita el proceso, por lo tanto este efecto es más predominante en relación a la agitación. A bajas temperaturas, con la viscosidad más elevada, el efecto de la agitación se sobrepone al de la temperatura.

Los autores Veglio y Beolchini (1997), concluyeron que la bioadsorción aumenta en la medida que se incrementa la temperatura (Say et al., 2003). Si se compara con los resultados obtenidos en este estudio se puede observar un comportamiento semejante cuando fue analizado el efecto de la temperatura en el aceite lubricante usado.

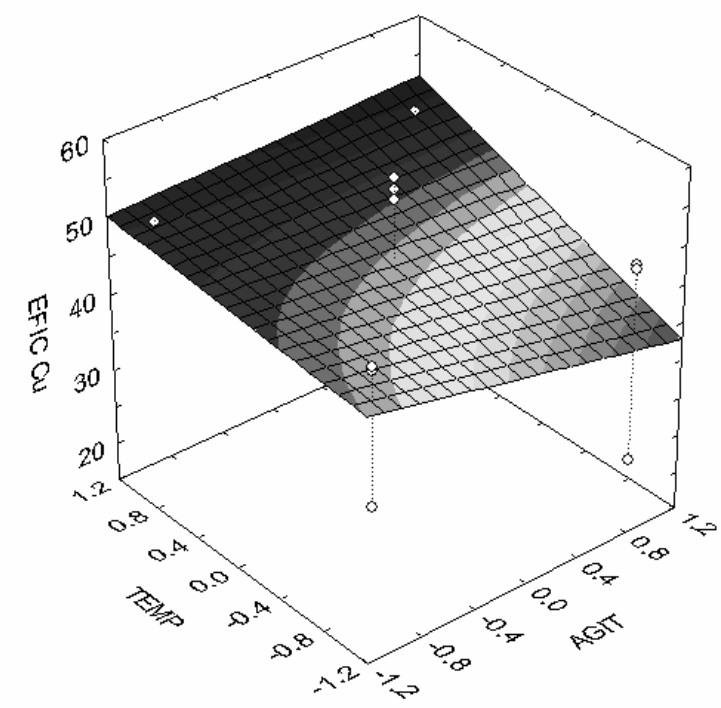

Fig. 7: Capacidad de remoción (eficiencia) del cobre en función de la agitación y de la temperatura

La capacidad de remoción (eficiencia) de cobre en función de la cantidad de biomasa (BM) protonada y de la concentración de $\mathrm{HCl}$ en la protonación son mostrados en la superficie de respuesta en la Fig. 8.
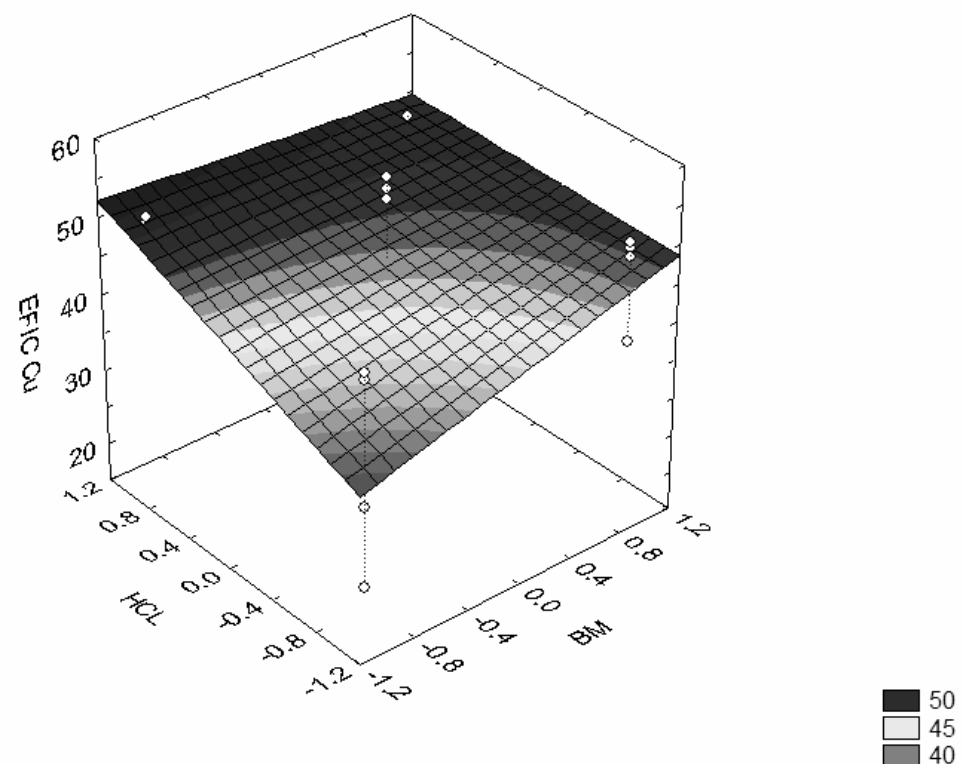

Fig. 8: Capacidad de remoción (eficiencia) del cobre en función de la cantidad de biomasa y concentración de HCL en la protonación 
En la Fig. 8 se puede observar, que para altas concentraciones de $\mathrm{HCl}$ se obtuvieron las mayores eficiencias. En el caso de bajas concentraciones de $\mathrm{HCl}$, se observa un aumento de la eficiencia con un aumento de la cantidad de biomasa. El HCl libera los sitios activos de la biomasa (BM), mejorando el proceso, por lo tanto al aumentar su concentración aumenta la eficiencia. Cuando esto no ocurre, se mejora la eficiencia aumentando la cantidad de la biomasa para que esta ofrezca más sitios activos en el proceso.

Los resultados del diseño experimental del estudio de remoción del hierro son mostrados en el gráfico de Pareto en la Fig. 9. La magnitud de cada efecto en esta figura esta representada por las columnas y líneas transversales con valor mayor o igual que $p=0,05$, lo que indica un significado estadístico del efecto de la variable o de su interacción con las otras.

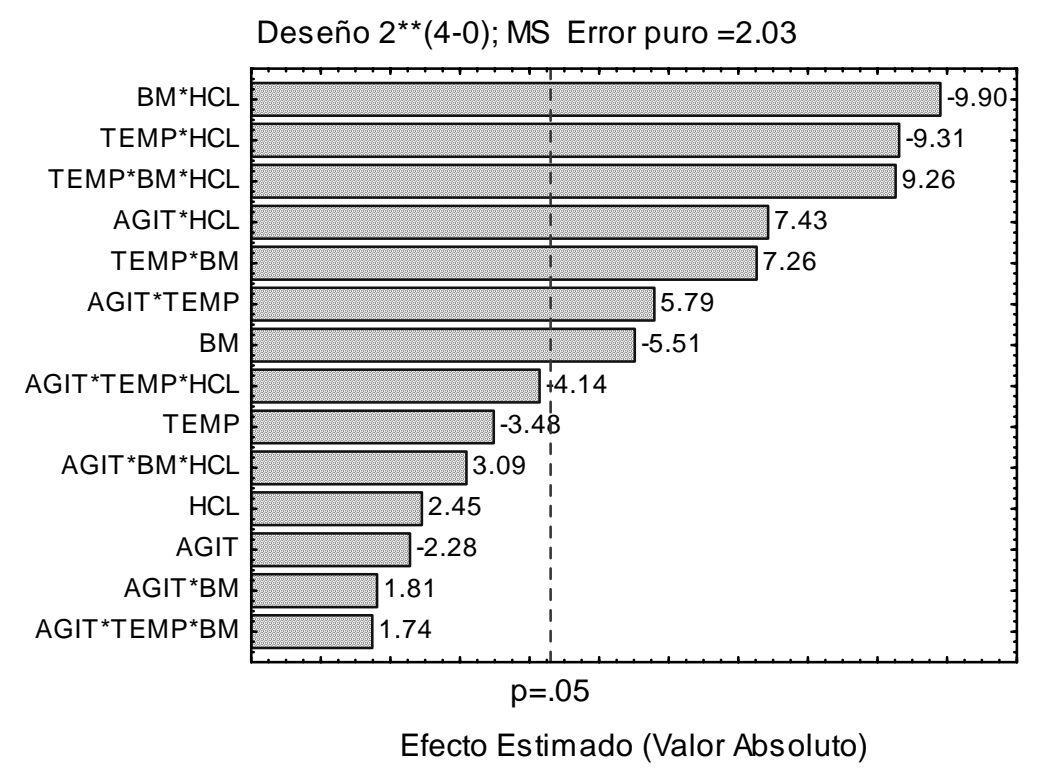

Fig. 9: Gráfico de Pareto para el estudio de la remoción del hierro

Analizándose el gráfico de Pareto se puede observar que para una confianza de 95\%, el efecto de la cantidad de biomasa (BM) y los efectos de interacción: $B M^{*} \mathrm{HCl}, T E M{ }^{*} \mathrm{HCl}, T E M{ }^{*} \mathrm{BM}{ }^{*} \mathrm{HCl}$, $A G I{ }^{*} \mathrm{HCl}, \mathrm{TEMP}^{\star} \mathrm{BM}$ y $A G T^{\star}{ }^{*}$ TEMP fueron estadísticamente significativos. El gráfico de los valores calculados versus los valores experimentales, para la remoción del hierro se muestran en la Fig. 10.

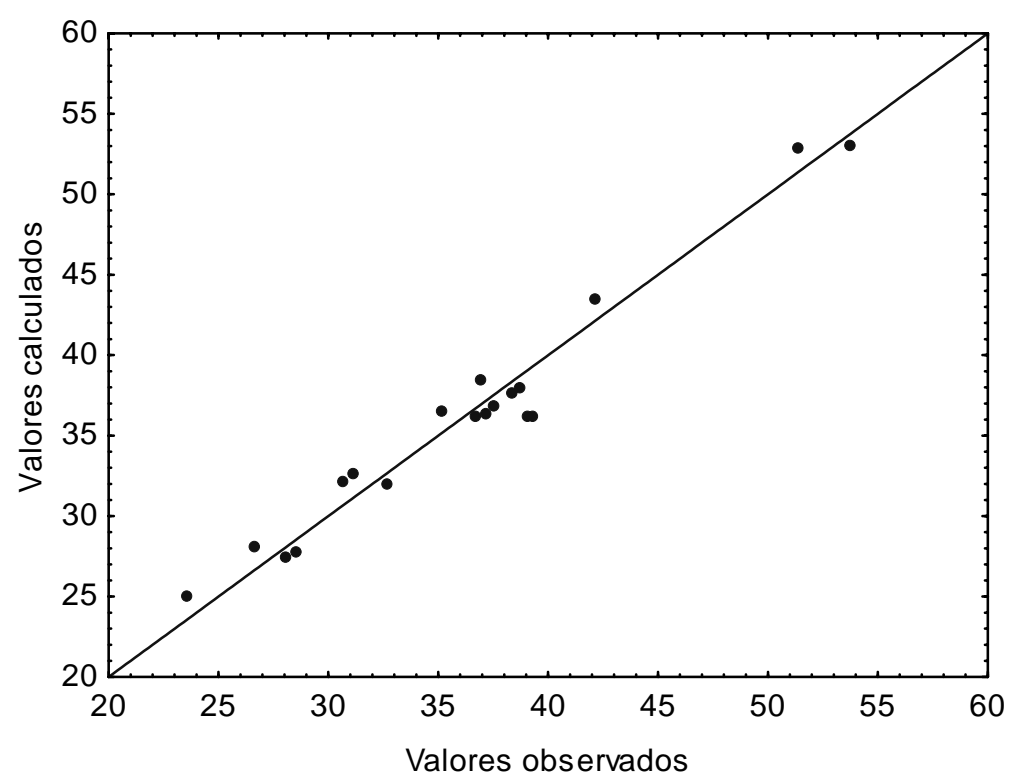

Fig. 10: Valores calculados en función de los valores observados para la remoción de hierro 
Se aprecia que los valores experimentales se aproximan satisfactoriamente a los valores calculados.

En la Fig. 11 se muestra la capacidad de remoción (eficiencia) de hierro en función de la agitación y temperatura. Se puede observar que las mayores eficiencias fueron obtenidas para una agitación y temperatura próximas de sus niveles mínimos. Una agitación menos vigorosa facilita el proceso, tanto en términos de tiempo de contacto como de la remoción del Fe, aumentando la eficiencia.

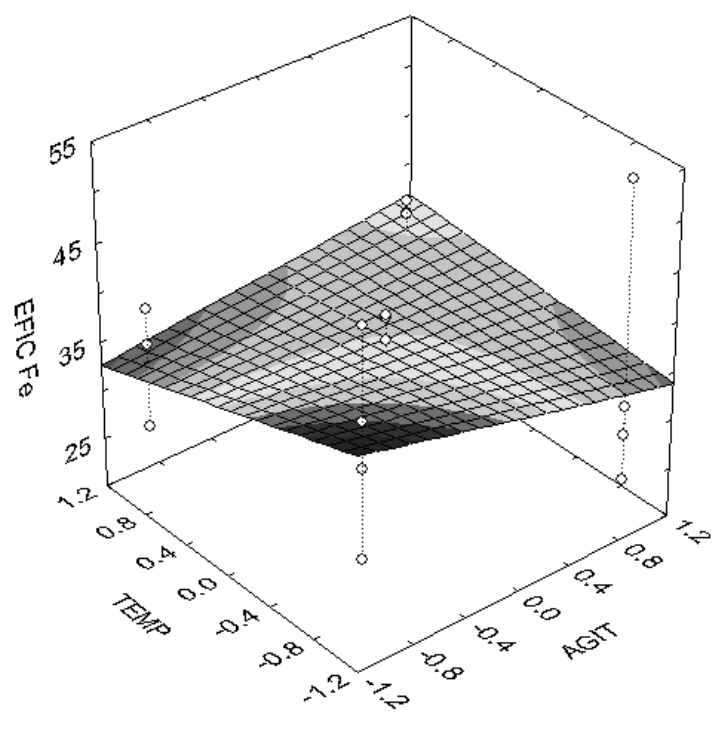

Fig. 11: Capacidad de remoción (eficiencia) del hierro en función de la agitación y de la temperatura

En la Fig. 12 se muestra la capacidad de remoción del hierro en función de la cantidad de biomasa (BM) y de la concentración de $\mathrm{HCl}$ en la protonación. Se puede observar que las mayores eficiencias fueron obtenidas para el nivel mínimo de la cantidad de biomasa y el nivel máximo de la concentración de $\mathrm{HCl}$. El efecto de la concentración de $\mathrm{HCl}$, que ayuda a liberar los sitios activos de la biomasa, supera el efecto del aumento de la cantidad de biomasa para disponer más sitios no liberados.

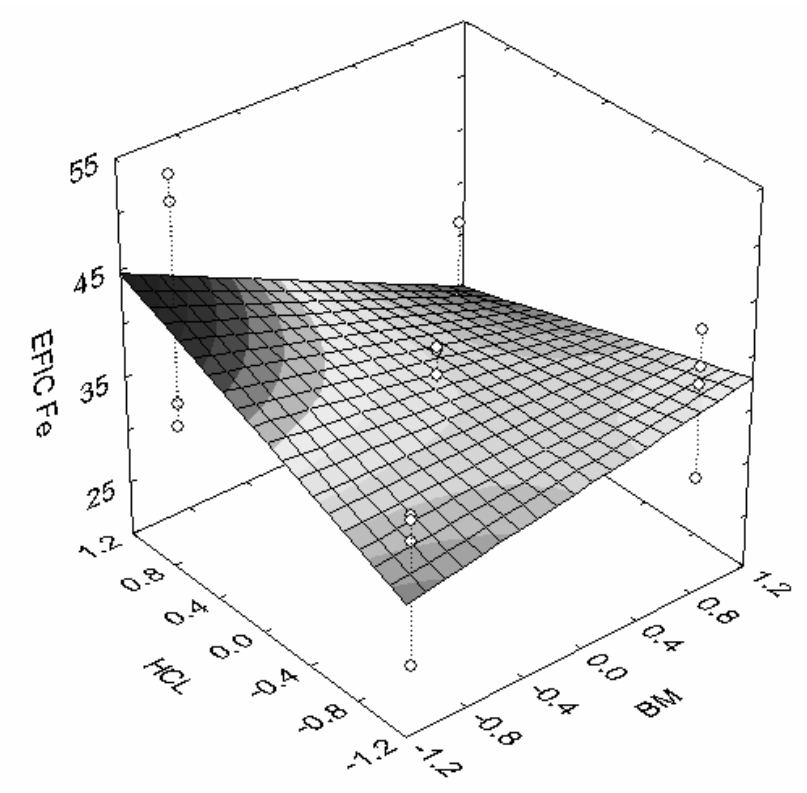

Fig. 12: Capacidad de remoción (eficiencia) del hierro en función de la cantidad de biomasa y de la concentración de $\mathrm{HCl}$ en la protonación 


\section{CONCLUSIONES}

De los resultados obtenidos se observó que la biomasa Sargassum sp. tiene un considerable potencial en la remoción del hierro y del cobre presentes en aceites lubricantes usados.

La protonación con el $\mathrm{HCl}$ libera los sitios activos de la biomasa, mejorando el proceso de remoción tanto del cobre como del hierro.

Las cinética de bioadsorción de hierro con la alga Sargassum sp. fue relativamente rápida. Para los iones cobrizos, por otro lado, no fue posible establecer un momento cuando el equilibrio se alcanza, aunque su cinética de bioadsorción también fue rápida.

Del análisis de las superficies de respuesta se concluye que el efecto de la temperatura es mayor que el de la agitación en la eficiencia de remoción de ambos metales. El aumento de la agitación solamente fue significativo en temperaturas bajas.

\section{AGRADECIMIENTOS}

A la "Agência Nacional de Petróleo (ANP)-PRH 14" por el apoyo financiero dado, y al programa de post graduación de Ingeniería Química de la UFRN (PPGEQ).

\section{REFERENCIAS}

Acosta, I., M.G. Moctezuma-Zárate, J. Cárdenas y C. Gutiérrez; Bioadsorción de cádmio (II) em solución acuosa por biomasas fúngicas, Información Tecnológica, 18(1), 9-14 (2007).

Alloway, B.J. y D.C. Ayres; Chemical Principles of Environmental Pollution. $1^{\mathrm{a}}$ Edição, Grã-Bretanha, Capítulo 2, 140-159 (1994).

Bailey, S.E., T.J. Olin, M. Bricka y D. Adrian; A review of potentially low-cost sorbents for heavy metals, Water Resouscer, 33(11), 2469-2479 (1999).

Barros Junior, L.M. Duarte, M.M.L; Macedo, G. R.; Remoção de metais pesados presentes nas águas de produção de campos de petróleo. Tesis de Magister, Dpto Eng. Química, UFRN, Natal, Brasil (2001).

Figueira M.N., B. Volesky, V.S.T. Ciminelli y F.A. Podick; Biosorption of metals in Brown seaweed biomass, Water Resource, 34(1), 196-204 (2004).

Gupta, G. y N. Torres; Use of fly ash in reducing toxicity of and heavy metals in wastewater effluent, J. of Hazardous Materials, Grã-Bretanha: Elsevier Science, 57(1), 243-248 (1998).

Hayashi, A.M.; Remoção de cromo hexavalente através de processos de biossorção em algas marinhas, Tesis de Titulación, Dpto Ing Química, UNICAMP, Campinas, Brasil (2001).

Kappor, A. у T. Viraraghavan; Fungal biosorption an alternative treatment option for heavy metal bearing wastewaters: a review, Bioresource Technology: 53, 195-206 (1995).

Kratochvil, D. y B. Volesky, Multicomponent biosorption in fixed beds, 34(12), Water Research, 31863196 (2000).

Kratochvil, D. y B. Volesky; Advances in the biosorption of heavy metal, Trends in Biotechnology, 16(7), 291-300 (1998).

Say, R., Y. Nalan y D. Adil; Biosorption of Cadmium, Lead, Mercury and Arsenic ions by the fungus Penicillium purpurogenum, Separation Science and Technology: 38(9), 2039-2053 (2003). 
Schmitt D., A. Muller, Z. Csogor, H. Fritz y C. Postem; The adsorption kinectics of metal ion sonto different microalgae and siliclous earth, Water Resource, 35(3), 779-785 (2001).

Tobin, J.M., D.G. Cooper y R.J. Neufeld; Uptake of metal íons by Rhizopus arrhizus biomass, Applied and Environmental Microbiology: 47(4), 821-824 (1984).

Veglio, F. y F. Beolchini; Removal of metals by biosorption: a review, Hydrometallurgy: 44(33), $301-$ 316 (1997).

Volesky, B.; Biosorbent, Behavior, Biosorption by fungal biomass, In Volesky, Biosorption of heavy metals, CRC Press, cap. 2-3, 139-172, U.S.A. (1990).

Yan, G. y T. Viraraghavan; Heavy-metal removal aqueous by fubgus Mucor rouxii, Water Research: 37(10), 4486-4496 (2003). 